\title{
Numerical simulation of magnetic blowout process of air arc plasma in electrical contacts*
}

\author{
by TASHIRO Shinichi**, ITODA Shuichi***, MORICHI Takahiro***, MURPHY B. Anthony**** and TANAKA \\ Manabu**
}

This study aims to clarify extinction phenomena of air arc plasma through magnetic blowout process in electrical contacts. For this purpose, numerical simulation taking into account ablation of polymer vapour was carried out. As a result, increase in the pressure due to polymer ablation was found to strongly affect the arc extinction phenomena. Especially, the pressure above 6 atm prevented arc re-ignition due to decrease in arc temperature which lowered electrical conductivity in the electrode gap.

Key Words: Air Arc Plasma, Electrical Contacts, Magnetic Blowout Process, Numerical Simulation

\section{Introduction}

The market for renewable energy such as solar power generators and electric vehicles is expanding against the background of increasing awareness of environmental issues such as global warming and soaring crude oil prices. In energy management equipment and battery control circuits used for them, since current interruption is necessary to prevent overcurrent due to short circuit, DC relays are mainly used from the viewpoint of high insulation and low resistance.

During the opening operation of the contacts in this relay, dielectric breakdown occurs between the contacts and the arc is ignited. Therefore, in order to cut off the current, it is indispensable to extinguish the arc instantaneously. If this arc continues for a long time, the inside of the casing is damaged and the contacts are consumed due to the high temperature arc. These can lead to serious accidents such as equipment damage and fire. In recent years, with the increase in voltage and current of devices, it is often required to cut off at a high voltage of several hundred volts or a large current of several hundred amperes. For this reason, it is important that the arc can be interrupted more safely and reliably than in the prior arts.

In electrical contacts, external magnetic field is generally used for an arc extinction. The application of the external magnetic field produces electromagnetic force for moving the arc, thus extending the arc length to increase the arc voltage ${ }^{1)}$. The contact of the extended arc to a polymer inner wall causes ablation of the polymer. The mixture of polymer vapour into the arc also induces the arc extinction 2). Although the outline of this series of

* Received: 2019.11.21, Presented at Visual-JW or WSE 2019

** Member, JWRI, Osaka University

*** Non-member, Omron Corporation

${ }^{* * * *}$ Non-member, CSIRO Manufacturing phenomena is known, due to the complexity of the phenomena and the difficulty of experimental observation, the detailed mechanism is still not clearly understood ${ }^{3)}$

This study aims to clarify extinction phenomenon of air arc plasma through magnetic blowout process in electrical contacts by conducting numerical simulation. In this paper, the result of the above numerical simulation carried out taking into account ablation of polymer is presented and a dominant factor to cause arc extinction is discussed.

\section{Simulation model}

Figure 1 shows a 3D simulation region with a width $X$ of $20 \mathrm{~mm}$, a length $Y$ of $20 \mathrm{~mm}$ and a height $Z$ of $42 \mathrm{~mm}$, which consists of a cathode region, an anode region and a gas region. The mesh size is non-uniform and approximately $0.1 \mathrm{~mm}$ at the minimum. The cathode and anode diameter is $10 \mathrm{~mm}$ and the length of those is 20 $\mathrm{mm}$. The electrode gap is $2 \mathrm{~mm}$. The simulation region is sealed with the polymer wall.

In addition, an external electrical circuit is defined. A constant voltage power supply, a resistor and electrical contacts are connected in series in the circuit. DC360 V is applied in the calculation. The resistance is $0.18 \Omega$. It leads to the maximum current of $2000 \mathrm{~A}$.

In practice, the arc phenomena in electric contacts are very complex for example due to influence of metal vapor evaporation, polymer ablation, increase in pressure, radiative energy transport and change in the thermodynamic and transport properties etc. However, a significantly simplified model is used in this simulation. In this simulation, the distributions of the temperature, flow, electrical potential, polymer vapour mole fraction etc. are obtained by solving time-dependent conservation equations ${ }^{4)}$. The SST (Menter's Shear Stress Transport) turbulence model is also 
used. The plasma is assumed to be optically thin. The thermodynamic and transport properties of both the air and POM are calculated as functions of temperature and pressure under the local thermodynamic equilibrium assumption ${ }^{5)}$. Those properties of the arc are obtained by weighting with the mole fraction of air and POM. Metal vapor evaporation from the electrodes is ignored. However, ablation of the polymer from the wall is taken into account. The polymer material is POM (polyacetal). The increase in pressure by polymer ablation and arc temperature rise is considered. In the initial condition, the simulation region is filled with air at $1 \mathrm{~atm}$. The electrical contact material is $\mathrm{Cu}$. The constant temperature of $1000 \mathrm{~K}$ is set inside the cathode and anode regions for simplicity. The temperature of the polymer wall is assumed to be $300 \mathrm{~K}$. The current is given inside the cathode region on the outer boundary and conducts to the bottom of the anode region where the potential is set to be 0 . The external magnetic field with a constant strength of $50 \mathrm{mT}$ is applied in the $X$ direction. The time step is $10^{-8} \mathrm{~s}$.

Mass conservation:

$\frac{\partial \rho}{\partial t}+\nabla \cdot(\rho \vec{u})=M_{P O M}$

Momentum conservation:

$\frac{\partial \rho \vec{u}}{\partial t}+\nabla \cdot(\rho \vec{u} \vec{u})=-\nabla p+\nabla \cdot \overrightarrow{\vec{\tau}}+\rho \vec{g}+\vec{j} \times \vec{B}$

Energy conservation:

$\frac{\partial \rho h}{\partial t}+\nabla \cdot(\rho h \vec{u})=\nabla \cdot(k \nabla T)+\vec{j} \cdot \vec{E}-Q_{r}$

Mass conservation of polymer vapour:

$\frac{\partial \rho Y}{\partial t}+\nabla \cdot(\rho Y \vec{u})=\nabla \cdot(\rho D \nabla Y)+M_{P O M}$

Current conservation:

$\nabla \cdot \sigma \nabla \Phi=0$

Ohm's law:

$\vec{j}=-\sigma \nabla \Phi=\sigma \vec{E}$

Vector potential:

$\nabla^{2} \vec{A}=-\mu_{0} \vec{j}$

Magnetic field:

$\vec{B}=\nabla \times \vec{A}$

Pressure:

$$
P=P_{0} \frac{m_{\text {ave }} T_{\text {ave }}}{m_{0} T_{0}}
$$

Evaporation flux of POM:

$F_{P O M}=A_{P O M} q^{B_{P O M}}$

where $\rho$ is the mass density, $\vec{u}$ is the velocity, $M_{\text {РОM }}$ is the source term relating to evaporation flux of POM from the wall which is given as a function of heat flux to the wall as presented at the end of this paragraph, $p$ is the pressure, $\overrightarrow{\vec{\tau}}$ is the viscosity, $\vec{g}$ is the gravity, $\vec{j}$ is the current density, $\vec{B}$ is the magnetic field, $h$ is the enthalpy, $k$ is the thermal conductivity, $T$ is the temperature, $\vec{E}$ is the electric field, $Q_{\mathrm{r}}$ is the arc radiation loss, $Y$ is the mass fraction of POM, $D$ is the diffusion coefficient of POM in air, $\sigma$ is the electrical conductivity, $\Phi$ is the electric potential, $\vec{A}$ is the vector potential, $\mu_{0}$ is the permeability of vacuum, $P$ is the averaged pressure at each time step referred for the calculation of the thermodynamic and transport properties. $P_{0}$ is the averaged pressure in the initial condition, $m_{\text {ave }}$ is the averaged mass density of the gas at each time step which is calculated taking into account an integrated value of $M_{\mathrm{POM}}$ over time, $T_{\text {ave }}$ is the averaged temperature at each time step, $m_{0}$ is the averaged mass density of the gas in the initial condition, $T_{0}$ is the averaged temperature in the initial condition. Even though the accuracy of $P$ described above is very coarse because of the strong assumption, influence of the pressure increase can be roughly evaluated. $F_{\mathrm{POM}}$ is the evaporation flux of POM and $q$ is heat flux to the wall. АРОм and B POM are constants. This was obtained by a similar approach with the literature ${ }^{6}$.

Table 1 shows the boundary conditions. The room temperature of $300 \mathrm{~K}$ is given on the outer boundary. The constant temperature of $1000 \mathrm{~K}$ is set inside the electrode for simplicity. The current is given inside the contact tip region on the top boundary and conducts to the bottom boundary where the potential is set to be 0 . The vector potential is 0 on the side boundary and the gradient of potential is 0 on the top and bottom boundaries.

In this paper, the following three cases are investigated for finding out the dominant factor to achieve arc extinction. Case 1: an arc extinction process without polymer ablation and increase in the pressure. Case 2: an arc extinction process with polymer ablation but without increase in the pressure. Case 3: an arc extinction process with polymer ablation and increase in pressure. The comparison between the case 1 and 2 gives information on influence of difference in the thermodynamic and transport properties of the air and POM on the arc extinction process. Fig. 2 shows thermal conductivity of air and POM. Tanaka et al reported 
that the arc extinction due to polymer ablation was considered to be mainly caused by higher thermal conductivity of polymer $(\mathrm{POM})^{7)}$. The comparison between the case 2 and 3 presents influence of the increase in the pressure, which is predicted to directly affect an energy balance of the arc.

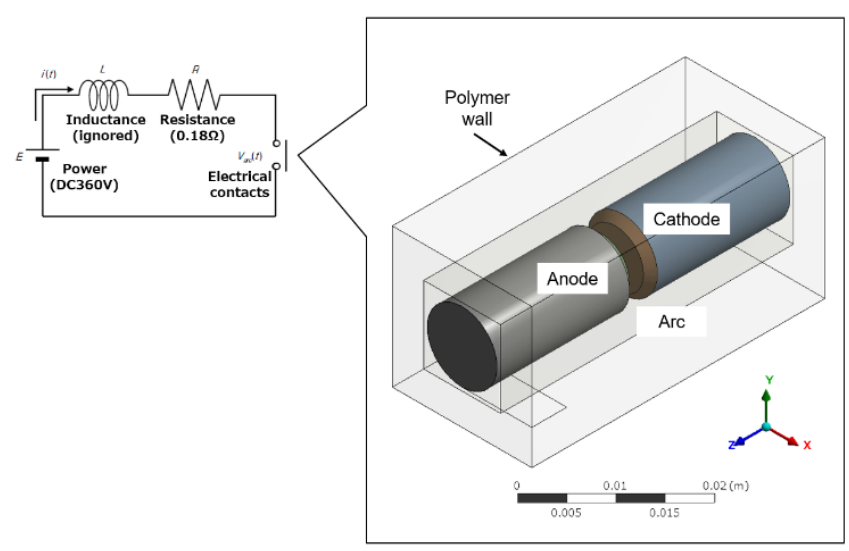

Fig. 1 A schematic diagram of simulation region.

Table 1 Boundary conditions

\begin{tabular}{|c|c|c|c|c|c|c|}
\hline \multicolumn{2}{|c|}{ Boundary } & Velocity & Ablation flux & Energy & Electric & Magnetic \\
\hline \multirow{2}{*}{ Top } & Electrode & - & - & $300 \mathrm{~K}$ & $-\sigma \partial \Phi / \partial n=j_{\text {given }}$ & $\partial A_{i} / \partial n=0$ \\
\hline & Wall & $\vec{u}=0$ & M POM & $300 \mathrm{~K}$ & $\partial \Phi / \partial n=0$ & $\partial A_{i} / \partial n=0$ \\
\hline Side & Wall & $\vec{u}=0$ & Mpom & $300 \mathrm{~K}$ & $\partial \Phi / \partial n=0$ & $A \mathrm{i}=0$ \\
\hline \multirow{2}{*}{ Bottom } & Electrode & - & Mpom & $300 \mathrm{~K}$ & $\Phi=0$ & $\partial A_{i} / \partial n=0$ \\
\hline & Wall & $\vec{u}=0$ & - & $300 \mathrm{~K}$ & $\partial \Phi / \partial n=0$ & $\partial A_{i} / \partial n=0$ \\
\hline
\end{tabular}

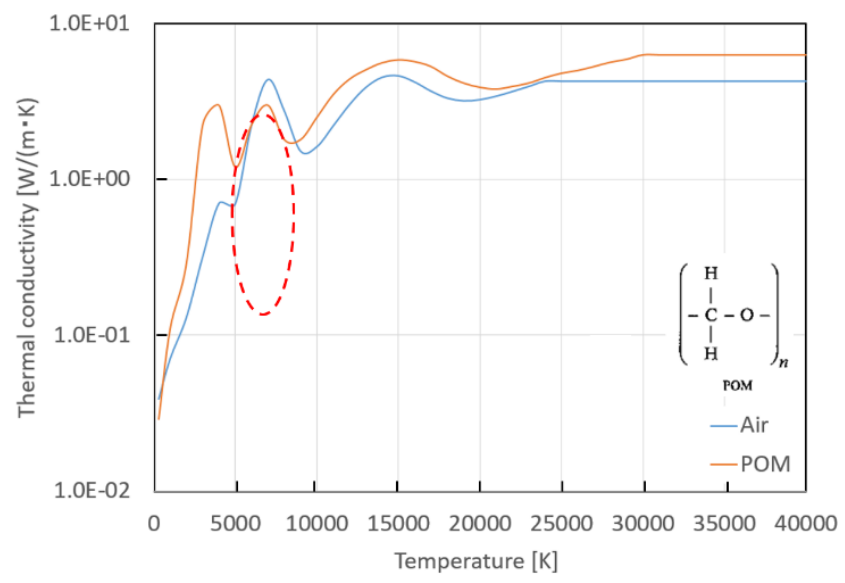

Fig. 2 Dependence of thermal conductivity on temperature.

\section{Result and discussion}

Figure 3 shows distributions of temperature and flow velocity vectors overwritten on mole fraction of polymer vapour on a Y-Z plane through arc axis and a X-Y plane through the middle height of gap at $\mathrm{t}=0 \mathrm{~s}$. Figure 4 shows time variation in arc voltage and current in case 1 . It was seen that the arc column was formed at the electrode center and the maximum arc temperature reached approximately $60000 \mathrm{~K}$. Immediately after the beginning of the calculation, the voltage and current were around $20 \mathrm{~V}$ and $1900 \mathrm{~A}$, respectively. After that, the arc was blown out leftward by the external magnetic field, leading to a slight increase in the voltage to $50 \mathrm{~V}$ and decrease in current to $1700 \mathrm{~A}$. This change indicates that the arc length increased after the arc length was extended after the arc passed through the contact corner. However, the arc extinction was not achieved.

Figure 5 shows time variation in arc voltage and current in case 2. After starting the calculation, the arc moved leftward due to the effect of the external magnetic field, then contacting with the polymer wall. However, the time variation in voltage and current was found to be almost similar with that in case 1. Also in this case, the arc extinction was not achieved. The result indicates that influence of difference in the thermodynamic and transport properties of the air and POM on the arc extinction process is thought to be small. This is because the higher thermal conductivity of POM is mainly obtained in a low temperature range. Accordingly, in case of a high current arc, this influence is considered to be negligible.

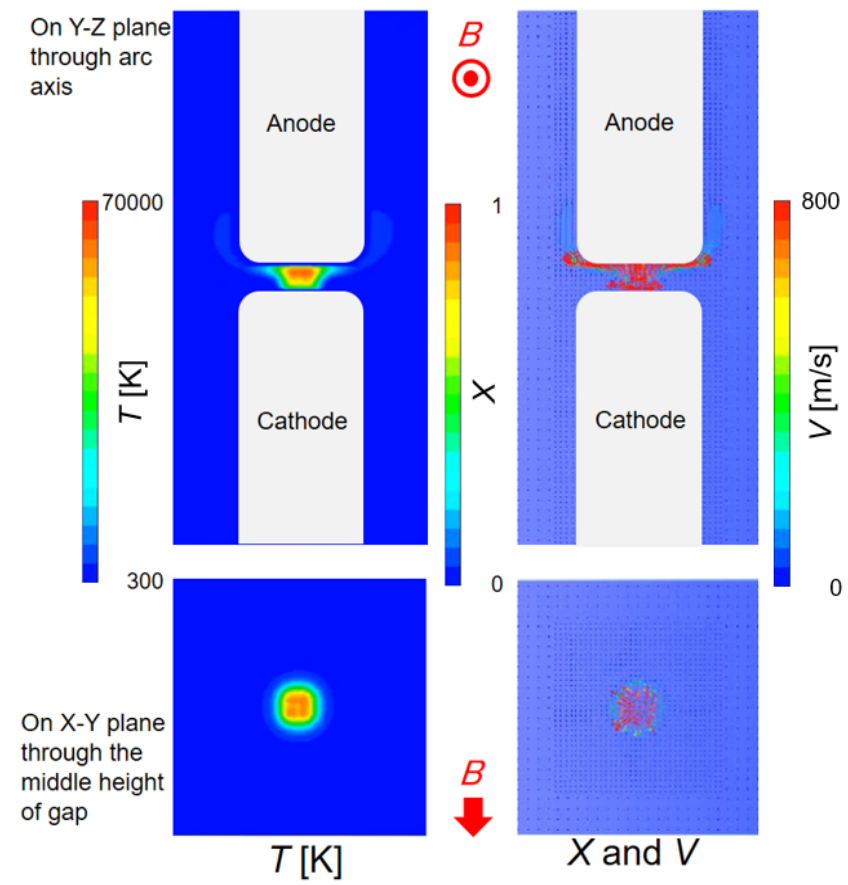

Fig. 3 Distributions of temperature (left) and velocity vectors overwritten on mole fraction of polymer vapour (right) at $t=0 \mathrm{~s}$ in case 1 


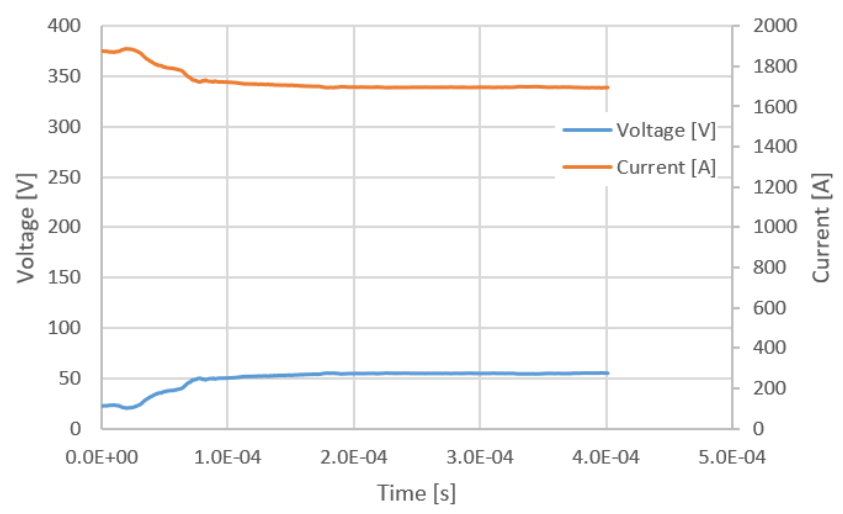

Fig. 4 Time variation in arc voltage and current in case 1.

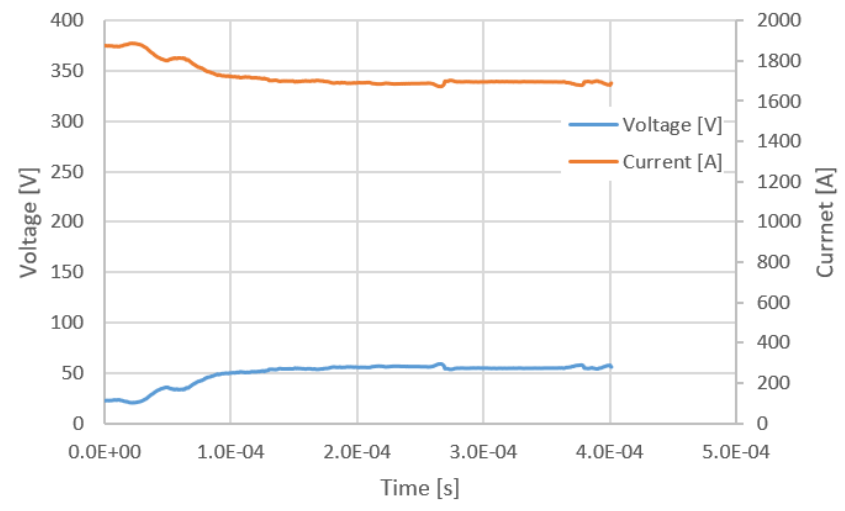

Fig. 5 Time variation in arc voltage and current in case 2.

Figure 6 shows distributions of temperature and flow velocity vectors overwritten on mole fraction of polymer vapour on a $\mathrm{Y}-\mathrm{Z}$ plane through arc axis and a X-Y plane through the middle height of gap at $\mathrm{t}=100 \mu \mathrm{s}$. The arc moved leftward due to the effect of the external magnetic field, then contacting with the polymer wall. At $\mathrm{t}=100 \mu \mathrm{s}$, the large amount of polymer vapour was found to be already evaporated from the wall to be mixed with the air. With this mixing, the arc temperature decreased to about $20000 \mathrm{~K}$. This temperature decrease is considered to be caused by increase in the radiation loss with increase in the pressure.

Figure 7 shows time variation in arc voltage and current. As seen in Fig. 6, the arc length was extended by applying the external magnetic field. Accordingly, the arc voltage gradually increased and eventually the current decreased with elapsed time. The several current oscillations seen around $\mathrm{t}=400 \sim 600 \mu$ s were caused by reignitions in the gap.

Figure 8 shows time variation in pressure and current. The pressure was seen to increase with time especially due to increase in the evaporation of polymer vapour, which induced lowering of the arc temperature through the enhanced arc radiation loss. The increase in pressure was found to strongly affect the arc extinction phenomena. When the pressure exceeded 6 atm, the arc extinction was completely achieved. Furthermore, the increase in pressure was found to contribute to prevent arc re-ignition due to decrease in arc temperature which lowered electrical conductivity in the electrode gap.

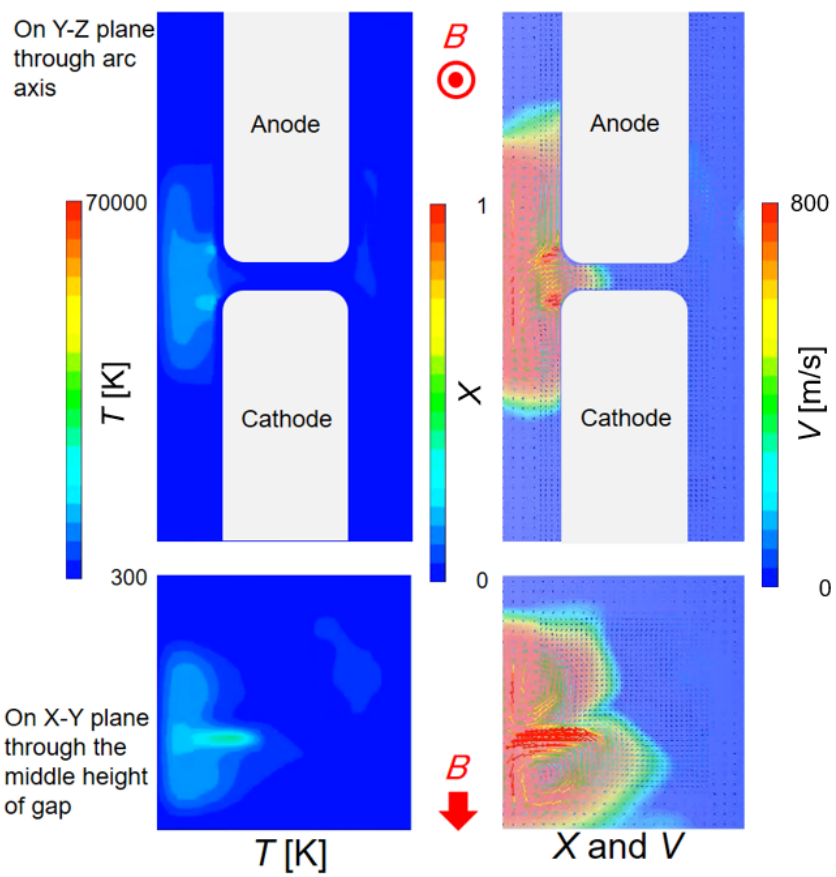

Fig. 6 Distributions of temperature (left) and velocity vectors overwritten on mole fraction of polymer vapour (right) at $t=100$ $\mu$ s in case 3 .

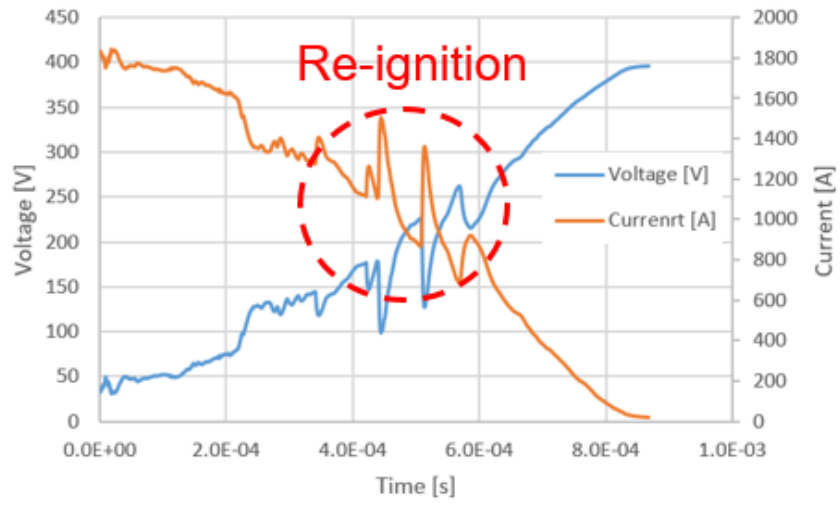

Fig. 7 Time variation in arc voltage and current in case 3.

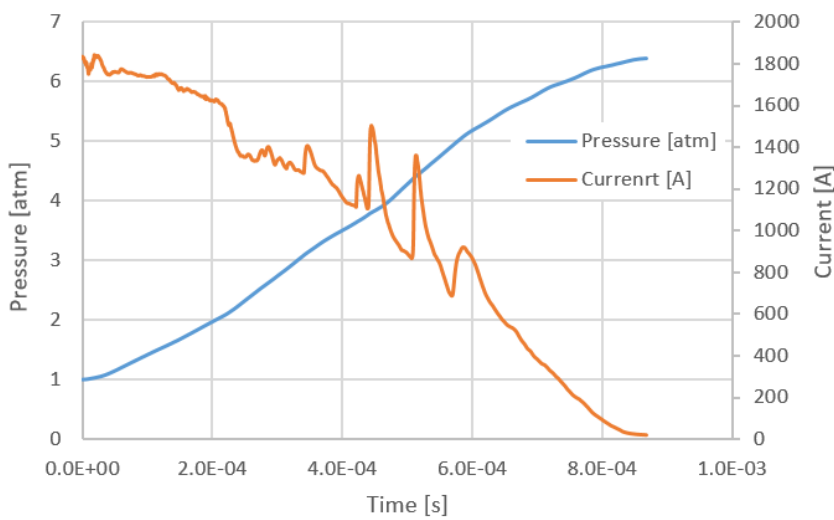

Fig. 8 Time variation in pressure and current in case 3. 


\section{Reference}

\section{Conclusions}

1) In order to clarify extinction phenomenon of air arc plasma through magnetic blowout process in electrical contacts, numerical simulation taking into account ablation of polymer vapour was carried out.

2) The pressure increased with time especially due to increase in the evaporation of polymer vapour, which induced lowering of the arc temperature through the enhanced arc radiation loss.

3) The increase in pressure was found to strongly affect the arc extinction phenomena. When the pressure exceeded $6 \mathrm{~atm}$, the arc extinction was completely achieved.

4) The increase in pressure contributed to prevent the arc reignition due to decrease in arc temperature which lowered electrical conductivity in the electrode gap.
1) H. Ohno et al: Travelling Characteristics of Electromagnetically Driven Arc Burning Between the Edge of Parallel Flat Electrodes in Atmospheric Pressure, T. IEE Japan 120-B (2000), 1504-1512.

2) Q. Ma et al: Simulation Study of the Influence of Wall Ablation on Arc Behavior in a Low-Voltage Circuit Breaker, IEEE TRANSACTIONS ON PLASMA SCIENCE, (2009) 261-269.

3) Fei Yang et al: Low-voltage circuit breaker arcs - simulation and measurements, J. Phys. D: Appl. Phys. 46 (2013), 273001 (19pp)1.

4) S. Tashiro, A. B. Murphy, M. Tanaka: Numerical simulation of fume formation process in GMA welding, Welding in the World, 62 (2018), 1331-1339.

5) M. Boulos, P Fauchais and E Pfender: THERMAL PLASMA, Plenum Pub. Corp. (1994), 159.

6) N. Osawa and Y. Yoshioka: Analysis of Nozzle Ablation Characteristics of Gas Circuit Breaker, IEEE Transactions on Power Delivery, 25 (2010), 755-761.

7) T. Onchi et al: Effect of Polymer Ablation Gas on Arc Quenching Properties around Current Zero, IEEJ Transactions on Power and Energy, 131 (2011), 609-620. 